\title{
Toxoplasmosis: Seroprevalencia y factores de riesgo en las majadas caprinas de la región noroeste de Argentina
}

\author{
Toxoplasmosis: risk factors in the goat flocks of \\ Argentina's Northwest region
}

\section{Toxoplasmose: fatores de risco em rebanhos caprinos da região noroeste da Argentina}

Suarez VH ${ }^{1}$, Martínez, GM"1 ; Dodero $A M^{2}$; Gos, $M L$

1 Estación Experimental Agropecuaria Salta, Instituto Nacional de Investigación Agropecuaria, RN 68, 4403, Cerrillos, Salta.

2 Instituto de Investigación Animal del Chaco Semiárido, Instituto Nacional de Investigación Agropecuaria, RN 68, 4403, Cerrillos, Salta.

3 Laboratorio de Inmunoparasitología Dto. de Epizootiología y Salud Pública Facultad de Ciencias Veterinarias, UNLP 60 y 118 La Plata.

Correo electrónico: suarez.victor@inta.gob.ar

\section{RESUMEN}

Se llevó a cabo una encuesta transversal en 54 unidades productivas familiares (UF) y 5 tambos caprinos comerciales (TC) en diferentes ecorregiones de Salta y Jujuy. El objetivo del trabajo fue estudiar la asociación entre la seroprevalencia de anticuerpos anti Toxoplasma gondii y aquellos factores relacionados con las unidades productivas y sus caprinos. Se procesaron sueros mediante la técnica de Inmunofluorescencia Indirecta $(n=629)$ y por ELISA indirecto $(n=619)$. Para el análisis estadístico de las variables se utilizó el chi-cuadrado, el Odds Ratio (OR) y la regresión logística. De las 59 majadas visitadas el $67,8 \%$ de ellas tuvo al menos un caprino seropositivo, mostrando disímil presencia entre las UF (66,7\%) y los TC (80\%). La prevalencia general fue del 18,2\% (95\% IC: 15,9-20,6\%). Los factores de riesgo significativamente asociados a una seroprevalencia de T. gondii mayor a un $20 \%$ de los caprinos muestreados fueron un porcentaje de gatos 
mayor al 1,1\% en relación con el total de cabras en servicio (OR 5,26; 95\% IC: 1,62-17,1) y la práctica del ordeñe (OR 13,03; 95\% IC: 2,19$80,3)$. Los presentes resultados muestran que las infestaciones por $T$. gondii en las majadas caprinas de Salta y Jujuy son importantes y ameritan profundizar los estudios.

Palabras clave: Toxoplasma gondii, seroprevalencia, factores de riesgo, noroeste de Argentina

\section{ABSTRACT}

A cross-sectional survey was performed in 54 family farm units (FU) and 5 dairy commercial farm (CF) from different regions of Jujuy and Salta provinces, with the aim of studying the association between the seroprevalence against Toxoplama gondii antibodies in goats flocks and risk factors related to the farm units and the goats management. Sera were processed by the indirect fluorescent antibody test IFAT (n $=629)$ and indirect ELISA $(\mathrm{n}=619)$ techniques. The Chi-square, Odds Ratio (OR) and logistic regression were used for the statistical analysis of the variables. Of the 59 visited flocks, $67.8 \%$ of them had at least one seropositive goat, showing differences between FU (66.7\%) and CF (80\%). The general prevalence was 18.2\% (95 \% CI: 15.9-20.6\%). The risk factors significantly associated to $T$. gondii prevalence greater than $20 \%$ of the sampled goats would be a percentage of cats greater than $1.1 \%$ in relation to the total number of mating goats (OR 5.26; 95\% CI: 1.62-17.1) and the milking practice (OR 13.03; 95\% CI: 2.1980.3). The present results show that infestations by T. gondii in the goat flocks of Salta and Jujuy are important and deserves further studies.

Key words: Toxoplasma gondii, seroprevalence, risk factors, Argentina's Northwest.

\section{Resumo}

Realizou-se um estudo transversal em 54 unidades familiares de produção (UF) e 5 tambos comerciais (TC) (propriedades leiteiras) em diferentes ecorregiões de Salta e Jujuy, com o objetivo de estudar a associação entre a soroprevalência de anticorpos anti Toxoplasma gondii em caprinos e fatores relacionados às unidades produtivas e seus animais. Os soros foram processados pela técnica de Imunofluorescência Indireta $(n=629)$ e ELISA indireto $(n=619)$. Para a análise estatística das variáveis foram utilizados o Qui-Quadrado, Odds Ratio (OR) e 
regressão logística. Dos 59 rebanhos visitados, 67,8\% deles apresentavam pelo menos um caprino soropositivo, apresentando presença diferente entre a UF $(66,7 \%)$ e os TC $(80 \%)$. A prevalência geral foi de 18,2\% (IC95\%: 15,9-20,6\%). Os fatores de risco significativamente associados a uma soroprevalência de T. gondii maior que $20 \%$ das cabras amostradas foi uma porcentagem maior que $1,1 \%$ em relação ao número total de cabras em serviço (OR 5,26; 95\% IC: 1,62-17,1) e a prática de ordenha (OR 13,03; 95\%IC: 2,19-80,3). Os presentes resultados mostram que as infestações por T. gondii nos rebanhos caprinos de Salta e Jujuy são consideráveis e merecem aprofundar os estudos.

Palavras-chave: Toxoplasma gondii, soroprevalência, fatores de risco, noroeste da Argentina.

Fecha de recepción artículo original: 09-03-2021

Fecha de aceptación para su publicación: 13-07-2021

\section{Introducción}

Toxoplasma gondii es un parásito protozoario de distribución mundial, que infecta a desde mamíferos hasta aves, incluyendo al hombre. $\mathrm{Su}$ importancia en medicina veterinaria y la producción de pequeños rumiantes se basa en mayor medida mayormente en que la primo infección durante la preñez puede inducir momificación fetal, abortos, nacimientos de crías débiles, pero también crías infectadas sanas. ${ }^{(1)}$ Las hembras portadoras latentes pueden abortar al quedar preñadas y también trasmitir en forma vertical esta parasitosis. ${ }^{(2)}$ Los felinos son los hospedadores definitivos ya que en ellos se produce el ciclo sexual y que la ingestión por otras especies de los ooquistes eliminados por sus heces es una de las principales fuentes de infestación. ${ }^{(3,4)}$

Esta parasitosis es de gran importancia en la salud pública, ya que está presente tanto en países subdesarrollados como en aquellos más desarrollados, estimándose que se encuentra en casi un tercio de la población mundial ${ }^{(5-7)}$. Aunque por lo general cursa en forma asintomática, en personas inmunodeprimidas puede causar problemas clínicos y durante los embarazos abortos o infestaciones congénitas de los recién nacidos debido a primo infestaciones o reactivación de los quistes preexistentes. ${ }^{(1)}$ Estudios en Córdoba muestran seroprevalencias en niños y en mujeres embarazadas del $51,7 \%$ y del $40,8 \%{ }^{(8)}$ y en otros trabajos realizados en hospitales de Buenos Aires en mujeres embarazadas del 17 al 51\%, estimándose que se estaría reduciendo su presencia a nivel de las grandes ciudades. ${ }^{(9)}$ 
Recientes estudios en San Luis y Buenos Aires Argentina muestran que su presencia en las majadas caprinas es importante. ${ }^{(10,11)}$ La provincia de Salta tampoco es la excepción ya que presenta una prevalencia general importante, comprometiendo no solo la producción animal sino también la salud de las familias que se ocupan de los caprinos. ${ }^{(12)}$

La crianza caprina ubicada generalmente en regiones marginales es de gran importancia para los pequeños productores hasta el punto de formar una fuerte asociación entre el hombre, la cabra y el medio ambiente. En la agricultura familiar del NOA también se ve reflejada esta asociación, donde la cabra además de producir leche y carne para el autoconsumo brinda a las familias la posibilidad de participar de las oportunidades económicas que otorga el creciente turismo y la afinidad por el queso de cabra que caracteriza a las poblaciones del noroeste argentino. ${ }^{(13)}$ Considerando que la forma de cría generalmente extensiva varía entre ecorregiones y que a esto se le suman explotaciones comerciales semi-intensivas con mayor adopción tecnológica, es importante tratar de identificar factores de riesgo que predispongan a una mayor presencia de este parásito en las majadas y su consecuente perjuicio en la salud de las familias productoras. ${ }^{(1,14)}$

Debido a todo lo expuesto y considerando la importancia de esta parasitosis, el propósito del presente trabajo fue investigar su seroprevalencia e identificar factores de riesgo asociados con la seroprevalencia de anticuerpos anti $T$. gondii en las majadas caprinas en diferentes ecorregiones de las provincias de Salta y Jujuy.

\section{Materiales y métodos}

\section{ÁREAS DE ESTUDIO}

Durante una visita a productores de 54 unidades familiares (UF) y 5 tambos comerciales (TC) que criaban caprinos en diferentes regiones de las provincias de Salta y Jujuy, se llevó a cabo una encuesta transversal. Las majadas relevadas estaban ubicadas en las ecorregiones de valles y quebradas áridas (VyQA, $n=33$ ), del chaco semiárido (CS, $n=$ 9) y de valles templados y pastizal serrano (VTyPS, n=17). Fig 1

\section{TIPO DE ENCUESTA Y CUESTIONARIO}

La encuesta se realizó por conveniencia, seleccionando aquellas unidades productivas, que estaban dispuestas a recibir en su predio la visita de los encuestadores. En cada visita la encuesta comprendió un 
cuestionario al productor, además de la toma de muestras de sangre a sus caprinos.

A partir de la respuesta de los productores se completó una encuesta, donde se registraron datos generales, de manejo y sanitarios referidos a sus majadas como: existencias caprinas, ovinas y bovinas, número de gatos, biotipo predominante, fuente de agua de bebida, tipo de servicio, número de cabritos destetados sobre cabras en servicio, sistema de manejo, suplementación, si ordeña, tasa de mortalidad, porcentaje de abortos, si realiza rutina de desparasitaciones e inoculación de minerales y si suministra sal.

\section{TOMA Y ANÁLISIS DE MUESTRAS}

Las muestras de sangre se tomaron abordando la vena yugular, un total de 1248, variaron de acuerdo con el total de caprinos, en promedio por majada estudiada fueron 21,1 muestras (máx.= 111 y mín.= 10). Las muestras fueron extraídas de hembras con más de un parto debido a que las cabras constituían numéricamente ala mayor parte de la majada. Seiscientos veinte y nueve sueros (30 majadas) se utilizaron para el diagnóstico de anticuerpos contra Toxoplasma gondii, se procesaron mediante la técnica de inmunofluorescencia indirecta (IFI), cuyo título de corte fue de $1 / 100$, de acuerdo al procedimiento descripto por Gos et al. (2017) y 619 sueros (29 majadas) fueron procesados mediante el test de ELISA indirecto (ID screen ${ }^{\circledR}$ Toxoplasmosis Indirect Multi-especies) de ID Vet Innovative Diagnosis, según las recomendaciones del fabricante para detectar anticuerpos específicos contra T. gondii. ; el punto de corte fue el porcentaje de reactividad $\geq 40=$ ODm - ODCNeg / ODCPos-ODCneg *100 considerando negativo a los menores o iguales al $40 \%$, dudosos entre 40 y $50 \%$ y positivos a los mayores o iguales al $50 \%$.

\section{Normas éticas:}

En todo momento se respetaron las normas bioéticas y los derechos de los animales durante los muestreos del ensayo.

\section{ANÁLISIS ESTADÍSTICO}

La majada se consideró positiva cuando se halló al menos un animal positivo, pero para determinar los factores de riesgo, se consideraron como casos positivos aquellas majadas con una prevalencia mayor o igual a un $20 \%$ y como casos negativos o bajos epidemiológicamente los de seroprevalencia nula o menores a un $20 \%$. Este valor se 
determinó a partir del promedio de seroprevalencia y para disminuir probables sesgos originados por el uso de las dos técnicas diferentes.

Todas las respuestas del cuestionario se incluyeron en el análisis estadístico univariado como variables independientes, utilizándose el Chi-cuadrado y el Odds Ratio (OR) para probar la asociación entre los casos positivos a T. gondii expuestos a las diferentes variables.

Las variables categóricas analizadas fueron ecorregión, sistema de manejo, fuente del agua de bebida, suplementación, biotipo, tipo de servicio, presencia de ovinos, presencia de bovinos, ordeña o no, aplica mineralizantes inyectables o en batea, si desparasita o no y la técnica utilizada: IFI o ELISA (Tabla 1). Las variables cuantitativas transformadas en categóricas fueron número de gatos/ total de cabras $\geq$ $1,1 \%$ o menor, total de cabras madres, porcentaje de abortos $\geq$ al $10 \%$ o menor, número de cabritos/cabras en servicio $\geq 85 \%$ o menor, tasa de mortalidad $\geq 15 \%$ o menor (Tabla 2 )

Aquellas variables que resultaron con un nivel de significancia del 0,15\% se las incluyó como regresoras en un análisis de regresión logística para determinar qué factores podrían considerarse predictores de una prevalencia a nivel majada de seropositividad a T. gondii $\geq 20 \%$.

La asociación de las variables categóricas con la presencia/ausencia de una seroprevalencia $\geq 20 \%$ de $T$ gondii fueron representadas e integradas gráficamente como puntos en el espacio mediante análisis de correspondencias múltiples. Los análisis se realizaron mediante el paquete estadístico de Info Stat y el nivel de significancia estadístico fue $\mathrm{p}<0,05$ (15).

\section{Resultados}

\section{CUESTIONARIO}

En promedio, la existencia caprina de las majadas fue $114,1 \pm 124,5$ animales (UF= 86,7 y TC= 315,8) sin tener en cuenta los cabritos menores de 2 meses de edad debido a que su número variaba de acuerdo a la época de las visitas. El número medio de hembras en servicio fue de $94,1 \pm 118,6$ (UF= 68,4 y TC= 286,5).

Un $68 \%$ de los productores criaban ovinos con sus caprinos, con un promedio de $18,4 \pm 17,3$ y solo un $38 \%$ tenían bovinos con un promedio de $33,6 \pm 24,0$. El $75 \%$ de productores tenían gatos con un número promedio de 2,2 $\pm 1,3$ por majada con un rango mínimo de 1 y un máximo de 5 gatos. El promedio del porcentaje de gatos con relación al promedio total de cabras en servicio fue de $2,86 \pm 3,91 \%$. 
El mayor porcentaje de las majadas $(47,6 \%)$ estaba compuesto por el biotipo Anglo Nubian y sus cruzas de base Criollo, había majadas con predominio de biotipos Criollos $(25,1 \%)$ y también sobre todo en los TC el biotipo predominante fue Saanen y sus cruzas con Criollo o Anglo Nubian $(27,3 \%)$.

Las cabras se proveían de agua mayormente de vertientes y acequias $(51 \%)$, directamente del río $(20 \%)$ o de pozos mediante bebederos $(29 \%)$.

Un $63 \%$ de los productores tenían un servicio continuo, mientras que los restantes practicaban un servicio estacionado y el promedio de cabritos por cabras al momento del servicio fue de $86 \pm 31$.

En cuanto al sistema de manejo, el 21,6\% practicaba un régimen semi intensivo y la mayoría restante un régimen extensivo $(78,4 \%)$. Al 58\% de las majadas se las suplementaba con grano, fardos u otros alimentos regionales alrededor del parto y en el ordeño o durante el invierno.

El 79,5\% de los productores visitados ordeñaba sus cabras.

En lo que se refiere a pérdidas productivas debido al porcentaje de caprinos muertos anualmente, un 51,2\% de los propietarios declararon una mortalidad superior al $15 \%$ y en lo referente a los abortos un $54,1 \%$ indicó que estos superaban en un año de parición el $10 \%$.

Solo en el $28,6 \%$ de las majadas se aplicaban en forma inyectable mineralizantes o sales en bateas y en un $76,7 \%$ se desparasitaba en forma rutinaria a todos los caprinos.

Serodiagnóstico

De las 59 majadas visitadas el 67,8\% de ellas tuvo al menos un caprino seropositivo, mostrando disímil presencia entre las UF $(66,7 \%)$ y los TC (80\%). La prevalencia media intra majadas fue de 20,14 \pm $23,7 \%$ seropositivos con extremos de 5,0 y $83,9 \%$ y la prevalencia general, es decir el número total de positivos sobre el total de sueros analizados fue del 18,2\% (95\% IC: 15,9-20,6\%). La figura 2 muestra el porcentaje de seropositivos a T. gondii y el número total de cabras en servicio de todas las majadas muestreadas discriminados por ecorregión.

Factores de riesgo analizados

La tabla 1 indica los resultados del análisis univariado de las variables categóricas y la tabla 2 los resultados de aquellas variables cuantitativas transformadas en categóricas.

A partir de los análisis univariados, los posibles factores de riesgos seleccionados fueron la presencia de gatos mayor al 1,1\% con relación al total de cabras en servicio, el ordeñe, el tipo de servicio, el sistema de manejo y los biotipos predominantes. El modelo de regresión logística 
al que fueron sometidas estas variables se presenta en la tabla 3. La función explicativa del análisis resultante muestra que el porcentaje de gatos mayor al $1,1 \%(\mathrm{p}<0,0061)$ y la práctica de ordeñe $(\mathrm{p}<0,087)$ serían los factores predictores con un margen de error menor al $10 \%$ asociados a una prevalencia mayor al $20 \%$ de anticuerpos de T. gondii en las majadas estudiadas.

El análisis multivariado de correspondencias múltiples que explica en un 49,2\% el posicionamiento espacial de las variables también muestra gráficamente una asociación entre el porcentaje de gatos mayor al 1,1 \% y el ordeñe con una infestación por T. gondii mayor al 20\% (Figura 3).

\section{Discusión}

Los presentes resultados evidencian la importancia de la toxoplasmosis en Salta y Jujuy, ya previamente descriptos por Dodero et al. (2019) ${ }^{(12)}$ y demuestran que la región NOA no es la excepción en el país ya que otras encuestas llevadas a cabo en otros sistemas de cría caprina también hallaron majadas seropositivas ${ }^{(16,10,11,17,18)}$

Cabe mencionar que el uso e inclusión de dos técnicas analíticas diferentes podría introducir sesgos. Sin embargo, ambas pruebas a pesar de su alta sensibilidad y especificidad (IFI 96 al 100\% y ELISA comercial $93-96 \%$ ) tienen factores para tener en cuenta como la subjetividad en la lectura del IFI y aunque validado en Europa, faltaría la validación local del ELISA importado. ${ }^{(19)}$ Para minimizar estos posibles sesgos y considerando que en un estudio de factores de riesgo importa que una majada este infectada dentro de ciertos parámetros determinados por la sensibilidad y especificidad, pero no tanto la prevalencia precisa de la misma es que se fijó el valor del promedio de prevalencia total, es decir del $20 \%$ de seropositivos en los análisis para ser asociados con los diferentes factores. Además de los análisis de sensibilidad y de estandarizar los posibles confusores, no se hallaron diferencias significativas en los valores de seroprevalencias entre ambas pruebas. ${ }^{(20-22)}$

Los resultados del presente trabajo, en principio a partir del análisis conjunto como en las estimaciones univariadas, muestran como principales factores de riesgo de infección con T. gondii a la presencia de gatos en un porcentaje mayor al 1,1\% en relación con el número total de cabras $\left(X^{2} 7,71 ; \mathrm{p}<0,005\right)$ y a la actividad de ordeñar las cabras $\left(X^{2} 8,11 ; \mathrm{p}<0,004\right)$.

En cuanto al número de gatos y su relación con el total de cabras, el análisis de momios o de ventajas comparativas estimó una chance 5 veces mayor $(\mathrm{OR} 5,26)$ de favorecer una mayor presencia de cabras 
seropositivas con un porcentaje alto de gatos. Una de las explicaciones es que independientemente del tipo de manejo o de explotación, la majada se encierra de noche, ${ }^{(23-24)}$ a veces en corrales muy reducidos, lo que propiciaría el posible contacto de las cabras con las deyecciones de los gatos domésticos. Además, los partos en los corrales favorecerían la infección de los gatos a partir del consumo de placentas o de fetos abortados. También se podría relacionar el pastoreo de las majadas en lugares donde hay felinos salvajes (pumas y gatos monteses) pero esta presencia es frecuente en la mayor parte de todas las regiones estudiadas. ${ }^{(23,25)}$

Estudios realizados en Brasil y en Países Bajos en caprinos similares a la presente investigación muestran una relación positiva entre un número mayor a 10 gatos ${ }^{(26)}$ o mayor a $1-4$ a $\geq$ de 5 gatos ${ }^{(27)}$ y la probabilidad de infección. También la simple presencia de gatos fue asociada a la prevalencia de anticuerpos para T. gondii en caprinos ${ }^{(28-32)}$ y ovinos. ${ }^{(33,34)}$ Contrariamente algunos trabajos no evidenciaron la presencia de gatos como factor de riesgo en rumiantes domésticos. ${ }^{(35-37)}$

El ordeñe resultó también como un factor de riesgo en los sistemas de producción caprina del NOA, con una probabilidad 13 veces mayor (OR 13,03) de favorecer un $\geq 20 \%$ de cabras seropositivas. Entre las explicaciones podríamos incluir que las cabras al ordeñarse se concentran más tiempo durante la mañana en los corrales hasta que terminan de ser ordeñadas y esto favorecería un mayor tiempo de contacto con las deyecciones de los gatos. También en lo que hace mayormente a las unidades familiares, que ordeñan directamente en los corrales de encierre, durante las visitas se observó la presencia de gatos bebiendo la leche de los primeros chorros que se arrojan al piso, posibilitando su infestación. ${ }^{(38)}$ Gos et al. $2017^{(11)}$, halló en tambos caprinos de la provincia de Buenos Aires seroprevalencias intra majadas que iban del 19,2 al 100\%.

El manejo de las majadas que solo involucró sistemas extensivos y semi-intensivos no tuvieron diferencias $(\mathrm{p}<0,12)$ como para ser considerados como factores de riesgo, así como el tipo de servicio $(\mathrm{p}<0,062)$, el tamaño de la majada $(\mathrm{p}<0,17)$, la suplementación $(\mathrm{p}<0,47)$, el biotipo racial $(0,11)$, la presencia de ovinos o bovinos $(p<0,81)$, la ecorregión $(\mathrm{p}<0,21)$ o la fuente de agua $(\mathrm{p}<0,27)$. Se podría pensar que un mayor riesgo se podría asociar a un manejo más intensivo, con servicios estacionados, suplementación o con una menor diversidad genética, sin embargo, el encierre nocturno practicado en todas estas regiones podría ser la razón que unificara las posibles diferencias. El encierre en corrales fue evidenciado como factor de riesgo de contraer T. gondii en el manejo de caprino, aunque asociado a la práctica de 
feedlot ${ }^{(39)}$. Los manejos extensivos de las UF comprendían aquellos que pastoreaban en los mismos predios todo el año y otros que realizaban trashumancia hacia los cerros en verano, ${ }^{(24,40)}$ pero tampoco se hallaron diferencias significativas entre ellos y entre las diferentes regiones visitadas $(\mathrm{p}<0,216)$. Neto et al. $(2008){ }^{(28)}$ en Brasil y Tzanidakis et al. (2012) ${ }^{(41)}$ en Grecia hallaron como factor de riesgo a los sistemas intensivos e semi intensivos respectivamente. También las majadas pequeñas y relacionadas con la crianza familiar, las fuentes de agua comunitarias, las razas puras o la presencia de bovinos han sido descriptas previamente como factor de riesgo ${ }^{(30,35,37,39,42,43)}$.

La dosificación rutinaria con mineralizantes y antihelmínticos al total de la majada no se asoció significativamente con mayores niveles de seroprevalencia en nuestro estudio, aunque algunos trabajos si evidenciaron como factores de riesgo a la falta de suplementación mineral. ${ }^{(28,44)}$ Posiblemente la suplementación mineral con los niveles de yodo indicados para regiones con carencias de este mineral como las quebradas y valles del NOA ${ }^{(25,45)}$ permitirían una mejor respuesta inmune frente a la infestación por T. gondii. ${ }^{(46)}$ La falta de asociaciones entre el índice de cabritos producidos o la tasa de abortos tendría su explicación en que muchas otras causas descriptas previamente tal vez más importantes estarían relacionadas a estos factores en las diferentes regiones del NOA. ${ }^{(47)}$

\section{Conclusiones}

Los presentes resultados muestran que las infecciones por T. gondii en las majadas caprinas en diferentes regiones de Salta y Jujuy presentan una prevalencia importante. La presencia de gatos en proporción al número de existencias y la práctica del ordeño serían factores de riesgo que predispondrían a las infestaciones con T. gondii en las majadas. Estos hallazgos preliminares que en mayor proporción se refieren a explotaciones familiares requieren estudios futuros donde se precise el verdadero riesgo sanitario a que estarían expuestas las personas y servirían de llamado de atención para que las autoridades llevaran a cabo campañas de prevención y concientización.

\section{Agradecimientos}

Los autores agradecen la desinteresada colaboración de las familias y dueños de las majadas caprinas. También se agradece al INTA por la financiación del presente trabajo. 


\section{Bibliografía}

1. Dubey JP. Toxoplasmosis of animal and humans. 2nd Edition. CRC Press. Boca Raton. FL. USA. 2010; 336 p.

2. Innes EA, Bartley PM, Buxton D, Katzer F. Ovine toxoplasmosis. Parasitology. 2009; 136:1887-1894. Cambridge University Press. doi:10.1017/S0031182009991636.

3. Dubey JP, Christie E, Pappas PW. Characterization of Toxoplasma gondii from the feces of naturally infected cats. Journal of Infectious Diseases. 1977; 136:432-435.

4. Jones JL, Dubey JP. Waterborne toxoplasmosis-recent developments. Experimental Parasitology. 2010; 124:10-25. doi.org/10.1016/j.exppara.2009.03.013

5. Abbas IE, Villena I, Dubey JP. A review on toxoplasmosis in humans and animals from Egypt. Parasitology. 2020; 147(2):135-159. doi:10.1017/s0031182019001367

6. Cook AJC, Gilbert RE, Buffolano W, Zufferey J, Petersen E, Jenum PA, et al. Sources of Toxoplasma infection in pregnant women: European multicentre case-control study. British Medical Journal. 2000; 321(7254):142-147.

7. Jones JL, Kruszon Moran D, Sanders Lewis K, Wilson M. Toxoplasma gondii infection in the United States, decline from the prior decade. American Journal of Tropical Medicine and Hygiene. 2007; 77:405-410.

8. Chiaretta AE, Sbaffo AM, Cristofolini AL, Molina MD. Estudio seroepidemiológico de la toxoplasmosis en niños de áreas de riesgo de la ciudad de Río Cuarto, Córdoba. Argentina. Parasitología. Latinoamericana. 2003; 58:112-117. doi.org/10.4067/ S0717-77122003000300004

9. Messina MT, Schneider V, Carral L, Outón E, Pari M, Rodríguez M, et al. Prevalencia de anticuerpos anti-Toxoplasma gondii en embarazadas en siete hospitales de Buenos Aires. Comparación entre 2006 y 2017. Medicina (Buenos Aires). 2019; 79(6):433-437.

10. Gos ML, Delgado MG, Bonzo EB, Arnonaga C, Pardini L, Unzaga JM, et al. Presencia de anticuerpos para Toxoplasma gondii y Neospora caninum en caprinos del departamento de Belgrano, provincia de San Luis, Argentina. XX Reunión Científico Técnica de la Asociación Argentina de Veterinarios de Laboratorios de Diagnóstico. 2014 nov 2729; Tucumán, Argentina.

11. Gos ML, Manazza JA, Späth EJA, Pardini L, Fiorentino MA, Unzaga JM, et al. Seroprevalence of Toxoplasma gondii and Neospora caninum infections in goats from two Argentinean provinces. Open Veterinary Journal. 2017; 7(4):319-322.

12 Dodero AM, Bertoni AE, Cortez HS, Salatin AO, Martínez Almudévar F, Gos ML, et al. Toxoplasmosis caprina en la provincia de Salta. Revista FAVE - S. Cs. Veterinarias. 2019; 18: 1-5; doi.org/10.14409/favecv.v18i1.7942

13. Martínez GM, Suarez VH. Lechería Caprina: producción, manejo, sanidad, calidad de leche y productos. 1ra Ed. INTA Ediciones, Colección Investigación, desarrollo e innovación. 2019; 167 p. (Consultado 10 ene 2021). Disponible en: https://inta.gob.ar/ documentos/lecheria-caprina-produccion-manejo-sanidad-calidad-de-leche.

14. Dubey JP, Verma,SK, Ferreira LR, Oliveira S, Cassinelli AB, Ying Y, et al. Detection and survival of Toxoplasma gondii in milk and cheese from experimentally infected 
goats. Journal of Food Protection. 2014; 77(10):1747-1753. doi:10.4315/0362-028X. JFP-14-167.

15. Di Rienzo JA, Casanoves F, Balzarini MG, Gonzalez L, Tablada M, Robledo CW. InfoStat, versión 2018, Grupo InfoStat, FCA, Universidad Nacional de Córdoba, Argentina. 2018.

16. Bedotti DO, Sánchez Rodríguez M. Observaciones sobre la problemática sanitaria del ganado caprino en el oeste Pampeano. Veterinaria Argentina. 2002; Vol. XIX(182):100-112.

17. Martínez A, Bincaz, J, Brihuega B, Sheridan M, Mozgovoj M, Parreño V, et al. Relevamiento sanitario en caprinos en una zona de peri-valle de la provincia de Río Negro, Argentina. Veterinaria Argentina. 2013; 303 julio (Consultado 21 nov 2020). Disponible en: www.veterinariargentina.com

18. Rossanigo CE, Venturini L, Venturini MC, Bacigalupe D, Unzaga JM. Toxoplasmosis caprina en majadas de San Luis. Reunión Científico Técnica de la Asociación Argentina de Veterinarios de Laboratorios de Diagnóstico, 2002 nov 14-15; Villa Gral. Belgrano, Córdoba.

19. Mangili PM, Vesco G, Feliziani F, Paoloni A, Menichelli M, Cagiola M, et al. Development and evaluation of the performance of an in-house ELISA to be used for the indirect diagnosis of Toxoplasmosis in sheep. SIDILV meeting, Parma, Italy. 2009; (Consultado 2 dic 2020). Disponible en: https://www.id-vet.com/pdfs/pdfs/TOXOS-MS/doc085. pdf

20. Groenwold RH, Sterne JA, Lawlor DA, Moons KG, Hoes AW, Tilling K. Sensitivity analysis for the effects of multiple unmeasured confounders. Ann. Epidemiol.. 2016; 26(9):605-611. doi:10.1016/j.annepidem.2016.07.009.

21. Gharibzadeh S, Mohammad K, Rahimiforoushani A, Amouzegar AM. Standardization as a Tool for Causal Inference in Medical Research. Arch Iran Med. 2016; 19. doi:0161909/aim.0011.

22. Sato T, Matsuyama Y. Marginal structural models as a tool for standardization. Epidemiology. 2003; 14(6):680-686. doi:10.1097/01.EDE.0000081989.82616.7d.

23. Suarez VH, Rosetto CB, Gaido AB, Salatin AO, Bertoni EA, Dodero AM, Viñabal AE, Pinto G, Brihuega BF, Romera SA, Maidana S. 2015. Prácticas de manejo y presencia de enfermedades en majadas caprinas de la región del chaco salteño. Veterinaria Argentina, 2015 diciembre (Consultado 7 ene 2021). Disponible en: www.veterinariargentina. com.

24. Suarez VH, Martínez GM, Olmos LH, Arapa C. Prácticas productivas de los sistemas familiares de cría caprina en los Valles Calchaquies (Payogasta, Salta) FAVE - Ciencias Agrarias. 2020; 19(1):97-110. doi 10.14409/a.v19i1.9456

25. Suarez VH, Dodero AM, Nievas JD, Martínez GM, Bertoni EA, Salatin AO, et al. Presencia de enfermedades en majadas caprinas de las quebradas áridas de Jujuy y Salta. Veterinaria Argentina. 2016 octubre (Consultado 7 ene 2021). Disponible en: www. veterinariargentina.com. 
26. Cavalcante A, Carneiro M, Gouveia A, Pinheiro R, Vitor RWA. Risk factors for infection by Toxoplasma gondii in herds of goats in Ceara', Brazil. Arquivo Brasileiro de Medicina Veterinária e Zootecnia. 2008; 60(1):36-41.

27. Deng H, Dam Deisz C, Luttikholt S, Maas M, Nielen M, Swart A, et al. Risk factors related to Toxoplasma gondii seroprevalence inindoor-housed dutch dairy goats. Preventive Veterinary Medicine. 2016; 124:45-51. doi.org/10.1016/j.prevetmed.2015.12.014

28. Neto JOA, Azevedo SS, Gennari SM, Funada MR, Pena HFJ, Araujo ARCP, et al. Prevalence and risk factors for anti-Toxoplasma gondii antibodies in goats of the Serido Oriental microregion, Rio Grande do Norte state, Northeast region of Brazil. Veterinary Parasitology. 2008; 156 (3-4):329-332

29. Garcia G, Sotomaior C, do Nascimento AJ, Navarro IT, Soccol VT. Toxoplasma gondii in goats from Curitiba, Paraná, Brazil: risks factors and epidemiology. Revista Brasileira de Parasitologia Veterinária. 2012; 21(1):42-47.

30. Nunes FVA, Vaez JR, Pinheiro RR, Cavalcante ACR, Vitor RWA, Ahid SMM. Soroprevalência e fatores associados à infecção por Toxoplasma gondii em caprinos de propriedades rurais do município de Mossoró, RN. Pesquisa Veterinária Brasileira. 2013; 33(5):565-570.

31. Liu ZK, Li JY, Pan H. Seroprevalence and risk factors of Toxoplasma gondii and Neospora caninum infections in small ruminants in China. Preventive Veterinary Medicine. 2015; doi.org/10.1016/j.prevetmed.2014.12.017

32. Bawm S, Maung WY, Win MY, Thu MJ, Chel HM, Khaing TA, et al. Serological survey and factors associated with Toxoplasma gondii infection in domestic goats in Myanmar. Hindawi Publishing Corporation Scientifica. 2016; ID 4794318. doi. org/10.1155/2016/4794318

33. Skjerve E, Waldeland H, Nesbakken T, Kapperud G. Risk factors for the presence of antibodies to Toxoplasma gondii in Norwegian slaughter lambs. Preventive Veterinary Medicine. 1998; 35(3):219-227.

34. Rêgo WMF., Paula NRO, Vitor RWA, Silva RAB, Diniz BLM, Sousa MM, et al. Risk factors for Toxoplasma gondii infection in goats and sheep raised in the State of Piauí in northeast Brazil. Small Ruminant Research. 2016; 141:17-23. doi:10.1016/j. smallrumres.2016.04.010

35. Gazzonis AL, Veronesi F, Di Cerbo AR, Zanzani SA, Molineri G, Morettaet I, et al. Toxoplasma gondii in small ruminants in Northern Italy - prevalence and risk factors. Annals of Agricultural and Environmental Medicine. 2015; 22(1):62-68.

36. Tilahun B, Tolossa YH, Tilahun G, Ashenafi H, Shimelis S. Seroprevalence and risk factors of Toxoplasma gondii infection among domestic ruminants in East Hararghe Zone of Oromia Region, Ethiopia. Veterinary Medicine International, 2018; 1-7, 4263470, doi.org/10.1155/2018/4263470

37. Abdallah MC, Kamel M, Karima B, Samir A, Djamel K, Rachid K et al. Cross-sectional survey on Toxoplasma gondii infection in cattle, sheep, and goats in Algeria: seroprevalence and risk factors Veterinary Science. 2019; 6(63):1-15. doi:10.3390/ vetsci6030063 
38. Mancianti F, Nardoni S, D'Ascenzi C, Pedonese F, Mugnaini L, Franco F, et al. Seroprevalence, detection of DNA in blood and milk and genotyping of Toxoplasma gondii in a goat population in Italy. BioMed Research International. 2013; (2):905326. doi: $10.1155 / 2013 / 905326$

39. Carneiro ACAV, Carneiro M, Gouveia AMG, Guimaraes AS, Marques APR, Vilas Boas LS, et al. Seroprevalence and risk factors of caprine toxoplasmosis in Minas Gerais, Brazil. Veterinary Parasitology. 2009; 160:225-229.

40. Suarez VH, Martínez GM, Nievas JD, Quiroga Roger J. Prácticas de manejo y producción en sistemas familiares de cría caprina en las quebradas áridas de Jujuy y Salta. Revista de Investigaciones Agropecuarias (INTA). 2017; 43(2):186-194.

41. Tzanidakis N, Maksimov P, Conraths FJ, Kiossis E, Brozos C, Sotiraki S, et al. Toxoplasma gondii in sheep and goats: seroprevalence and potential risk factors under dairy husbandry practices. Veterinary Parasitology, 2012; 190:340-348 doi.org/10.1016/j. vetpar.2012.07.020

42. Gebremedhin EZ, Agonafir A, Tessema TS, Tilahun G, Medhin G, Vitale M, et al. Seroepidemiological study of ovine toxoplasmosis in East and West Shewa Zones of Oromia Regional State, Central Ethiopia. BCM Veterinary Research. 2013; 9:117. (Consultado 23 jun 2020). Disponible en: http://www.biomedcentral.com/1746-6148/9/117

43. Deksne G, Ligere B, Šneidere A, Jokelainen P. Seroprevalence and Factors Associated with Toxoplasma gondii Infections in sheep in Latvia: Latvian Dark Headed sheep breed associated with higher seroprevalence. Vector Borne Zoonotic Diseases. Mary Ann Liebert, Inc. 2017. doi: 1010.1089/vbz.2016.2003

44. Lopes WD, Santos TR, da Silva RS, Rossanese WM, de Souza FA, Faria Rodrigues JD, et al. Seroprevalence of and risk factors for Toxoplasma gondii in sheep raised in the Jaboticabal microregion, Sao Paulo State, Brazil. Research Veterinary Science. 2010; 88:104-106.

45. Suarez VH, Martínez GM, Bertoni EA, López Linares S. Respuesta al tratamiento con una formulación en base a yodo de cabras Saanen en el noroeste argentino. Resúmenes XXVI ALPA y V Simposio Internacional de Producción Animal, 2018 mayo 28-31; Guayaquil, Ecuador.

46. Slosárková S, Literaâk I, SkrÏivaânek M, Svobodová V, Suchya P, Herzig I. Toxoplasmosis and iodine deficiency in Angora goats. Veterinary Parasitology. 1999; 81:89-97.

47. Suarez VH, Martínez GM. Infectious and parasitic disease presence in smallholder's dairy goat flocks from the arid regions of Northwestern Argentina. Dairy and Veterinary Science Journal. 2019; 14(3):555886. doi: 10.19080/JDVS.2019.14.555886 


\section{Tablas}

Tabla 1: Factores de riesgo asociados a majadas con una seroprevalencia de anticuerpos contra Toxoplasma gondii mayor a un 20\%, según resultados de los análisis univariados de las variables categóricas $(\mathrm{p}<0,05) . X^{2}$ : Chi cuadrado, $P: \mathrm{p}$ valor, OR: odds ratio, LI: Límite inferior, LS: límite superior.

\begin{tabular}{|c|c|c|c|c|c|c|}
\hline Variable & $\begin{array}{l}\text { Nivel de } \\
\text { variable }\end{array}$ & $\begin{array}{l}\text { T. gondii } \\
\quad \geq 20\end{array}$ & $\begin{array}{l}\text { T. gondii } \\
\leq 19,9\end{array}$ & $\begin{array}{l}X^{2} \\
\mathbf{P}\end{array}$ & OR & $\begin{array}{l}\text { LI } 95 \% \\
\text { LS } 95 \%\end{array}$ \\
\hline \multirow{3}{*}{ Ecorregión* } & VyQA & 16 & 17 & \multirow{3}{*}{$\begin{array}{c}3,06 \\
0,216\end{array}$} & & \\
\hline & $\mathrm{ChS}$ & 1 & 8 & & & \\
\hline & VTyPS & 6 & 11 & & & \\
\hline \multirow{2}{*}{$\begin{array}{c}\text { Sistema de } \\
\text { Manejo }\end{array}$} & Semi intensivo & 8 & 5 & \multirow{2}{*}{$2,4 \quad 0,121$} & \multirow{2}{*}{2,91} & 0,77 \\
\hline & Extensivo & 15 & 26 & & & 11 \\
\hline \multirow{2}{*}{ Suplementación } & Si suplementa & 14 & 15 & \multirow{2}{*}{$\begin{array}{c}0,51 \\
0,474\end{array}$} & \multirow{2}{*}{1,51} & 0,5 \\
\hline & No suplementa & 8 & 14 & & & 4,64 \\
\hline \multirow{3}{*}{ Biotipo* } & AN y cruzas & 11 & 13 & \multirow{3}{*}{$\begin{array}{c}10,38 \\
0,11\end{array}$} & & \\
\hline & Criolla & 1 & 12 & & & \\
\hline & SA, AN y cruzas & 5 & 9 & & & \\
\hline \multirow{2}{*}{ Servicio } & Continuo & 22 & 12 & \multirow{2}{*}{$\begin{array}{c}3,48 \\
0,062\end{array}$} & \multirow{2}{*}{3,03} & 0,96 \\
\hline & Estacionado & 8 & 11 & & & 9,55 \\
\hline \multirow{2}{*}{$\begin{array}{c}\text { Presencia de } \\
\text { ovinos }\end{array}$} & No & 12 & 13 & \multirow{2}{*}{$\begin{array}{c}0,87 \\
0,351\end{array}$} & \multirow{2}{*}{0,59} & 0,2 \\
\hline & $\mathrm{Si}$ & 10 & 17 & & & 1,76 \\
\hline \multirow{2}{*}{$\begin{array}{l}\text { Presencia de } \\
\text { bovinos }\end{array}$} & No & 14 & 17 & \multirow{2}{*}{$0,130,71$} & \multirow{2}{*}{0,81} & 0,27 \\
\hline & $\mathrm{Si}$ & 8 & 14 & & & 2,47 \\
\hline \multirow{3}{*}{ Fuente de agua } & Vertiente & 11 & 12 & \multirow{3}{*}{$5,190,27$} & & \\
\hline & Río & 3 & 6 & & & \\
\hline & Pozo & 2 & 12 & & & \\
\hline \multirow{2}{*}{ Ordeñan } & $\mathrm{Si}$ & 21 & 19 & \multirow{2}{*}{$8,110,004$} & \multirow{2}{*}{13,03} & 2,19 \\
\hline & No & 1 & 12 & & & 80,3 \\
\hline \multirow{2}{*}{$\begin{array}{c}\text { Aplican } \\
\text { mineralizantes }\end{array}$} & No & 17 & 18 & \multirow{2}{*}{$1,850,17$} & \multirow{2}{*}{2,5} & 0,69 \\
\hline & $\mathrm{Si}$ & 4 & 10 & & & 9,06 \\
\hline \multirow{2}{*}{$\begin{array}{c}\text { Aplican } \\
\text { antihelmínticos }\end{array}$} & No & 5 & 8 & \multirow{2}{*}{$0,290,59$} & \multirow{2}{*}{1,5} & 0,36 \\
\hline & $\mathrm{Si}$ & 15 & 18 & & & 6,19 \\
\hline \multirow{2}{*}{ Técnica } & IFI & 12 & 19 & 20 & קOP & 0,34 \\
\hline & ELISA & 11 & 17 & $0,000,02$ & 0,90 & 2,78 \\
\hline
\end{tabular}

* VyQA: valles y quebradas áridas; ChS: chaco semiárido; VTyPS: valles templados y pastizales serranos

* AN: Anglo Nubian; SA: Saanen 
Tabla 2: Factores de riesgo para majadas con una seroprevalencia de anticuerpos contra Toxoplasma gondii mayor a un 20\%, según resultados mediante análisis univariado de variables cuantitativas $(\mathrm{p}<0,05) . X^{2}$ : Chi cuadrado, $P$ : $\mathrm{p}$ valor, OR: odds ratio, LI: Límite inferior, LS: límite superior.

\begin{tabular}{|c|c|c|c|c|c|c|}
\hline Variable & $\begin{array}{l}\text { Nivel de } \\
\text { variable }\end{array}$ & $\begin{array}{l}\text { T. gondii } \\
\geq 20\end{array}$ & $\begin{array}{l}\text { T. gondit } \\
\leq 19,9 \\
\end{array}$ & $\begin{array}{l}X^{2} \\
P \\
\end{array}$ & OR & $\begin{array}{l}\text { LI } 95 \% \\
\text { LS } 95 \% \\
\end{array}$ \\
\hline \multirow{2}{*}{$\begin{array}{c}\mathrm{N}^{\circ} \text { de gatos } / \mathrm{n}^{\circ} \\
\text { total de cabras } \\
\text { en servicio } \\
\end{array}$} & Gatos $\geq 1,1 \% *$ & 19 & 13 & \multirow{2}{*}{$\begin{array}{c}7,71 \\
0,005\end{array}$} & \multirow{2}{*}{5,26} & 1,62 \\
\hline & Gatos $\leq 1 \%$ & 5 & 18 & & & 17,1 \\
\hline \multirow{2}{*}{$\begin{array}{c}\mathrm{N}^{\circ} \text { total de ca- } \\
\text { bras en servicio }\end{array}$} & $\leq \operatorname{de} 45$ & $\begin{array}{l}11 \\
\ldots \ldots\end{array}$ & 9 & \multirow{2}{*}{$\begin{array}{l}1,89 \\
0,17 \\
\end{array}$} & \multirow{2}{*}{2,22} & $\begin{array}{l}0,72 \\
\ldots \ldots \ldots\end{array}$ \\
\hline & $\geq \operatorname{de} 46$ & 11 & 22 & & & 6,83 \\
\hline \multirow{2}{*}{$\begin{array}{c}\text { Porcentaje de } \\
\text { abortos }\end{array}$} & $\geq \operatorname{de} 10 \%$ & 10 & 12 & \multirow{2}{*}{$\begin{array}{l}0,03 \\
0,85\end{array}$} & \multirow{2}{*}{0,89} & 0,25 \\
\hline & $\leq$ de $9,9 \%$ & 8 & 9 & & & 3,13 \\
\hline \multirow{2}{*}{$\begin{array}{c}\mathrm{N}^{\circ} \text { de cabritos } / \\
\mathrm{n}^{\circ} \text { de cabras en } \\
\text { servicio }\end{array}$} & $\leq \operatorname{de} 85 \%$ & 10 & 13 & \multirow{2}{*}{$\begin{array}{l}0,32 \\
0,57\end{array}$} & \multirow[b]{2}{*}{1,41} & 0,44 \\
\hline & $\geq$ de $86 \%$ & 9 & 14 & & & 4,56 \\
\hline \multirow{2}{*}{$\begin{array}{c}\text { Tasa de } \\
\text { Mortalidad } \\
\text { total } \\
\end{array}$} & $\geq \operatorname{de} 15 \%$ & 10 & 14 & \multirow{2}{*}{$\begin{array}{l}1,31 \\
0,26\end{array}$} & \multirow{2}{*}{2,08} & 0,61 \\
\hline & $\leq$ de $15 \%$ & 6 & 15 & & & 7,13 \\
\hline
\end{tabular}

Tabla 3: Modelo de regresión logística del estatus de presencia de seropositividad a T. gondii $\geq 20 \%$. b: constante b, EE: error estándar, $\mathrm{Chi}^{2}$ : Chi cuadrado, OR: odds ratio, LI: Límite inferior, LS: límite superior.

\begin{tabular}{|c|c|c|c|c|c|c|c|c|}
\hline Variables & $\begin{array}{c}\text { Nivel de } \\
\text { variable }\end{array}$ & b & E.E. (b) & O.R. & $\begin{array}{c}\text { Wald } \\
\text { LI(95\%) }\end{array}$ & $\begin{array}{c}\text { Wald } \\
\text { LS(95\%) }\end{array}$ & $\begin{array}{c}\text { Wald } \\
\text { Chi }^{2}\end{array}$ & p-valor \\
\hline Constante & & 2,79 & 1,86 & 16,32 & 0,43 & 625,9 & 2,25 & 0,134 \\
\hline Gatos: $\geq 1,1 \%$ & $\mathrm{Si} /$ no & 1,36 & 0,72 & 3,99 & 0,99 & 16,01 & 3,52 & 0,061 \\
\hline Ordeña & $\mathrm{Si} /$ no & $-1,66$ & 1,15 & 0,22 & 0,02 & 1,98 & 2,93 & 0,087 \\
\hline Raza & $\begin{array}{c}\text { AN y cz / Criolla } \\
\text { / Sa, AN y cz }\end{array}$ & $-0,44$ & 0,4 & 0,64 & 0,29 & 1,4 & 1,23 & 0,267 \\
\hline Sistema & $\begin{array}{c}\text { Semi intensivo/ } \\
\text { extensivo }\end{array}$ & $-0,83$ & 0,81 & 0,44 & 0,09 & 2,14 & 1,04 & 0,308 \\
\hline Servicio & $\begin{array}{c}\text { Continuo / } \\
\text { estacionado }\end{array}$ & $-0,29$ & 0,68 & 0,75 & 0,2 & 2,84 & 0,18 & 0,668 \\
\hline
\end{tabular}




\section{Figuras}

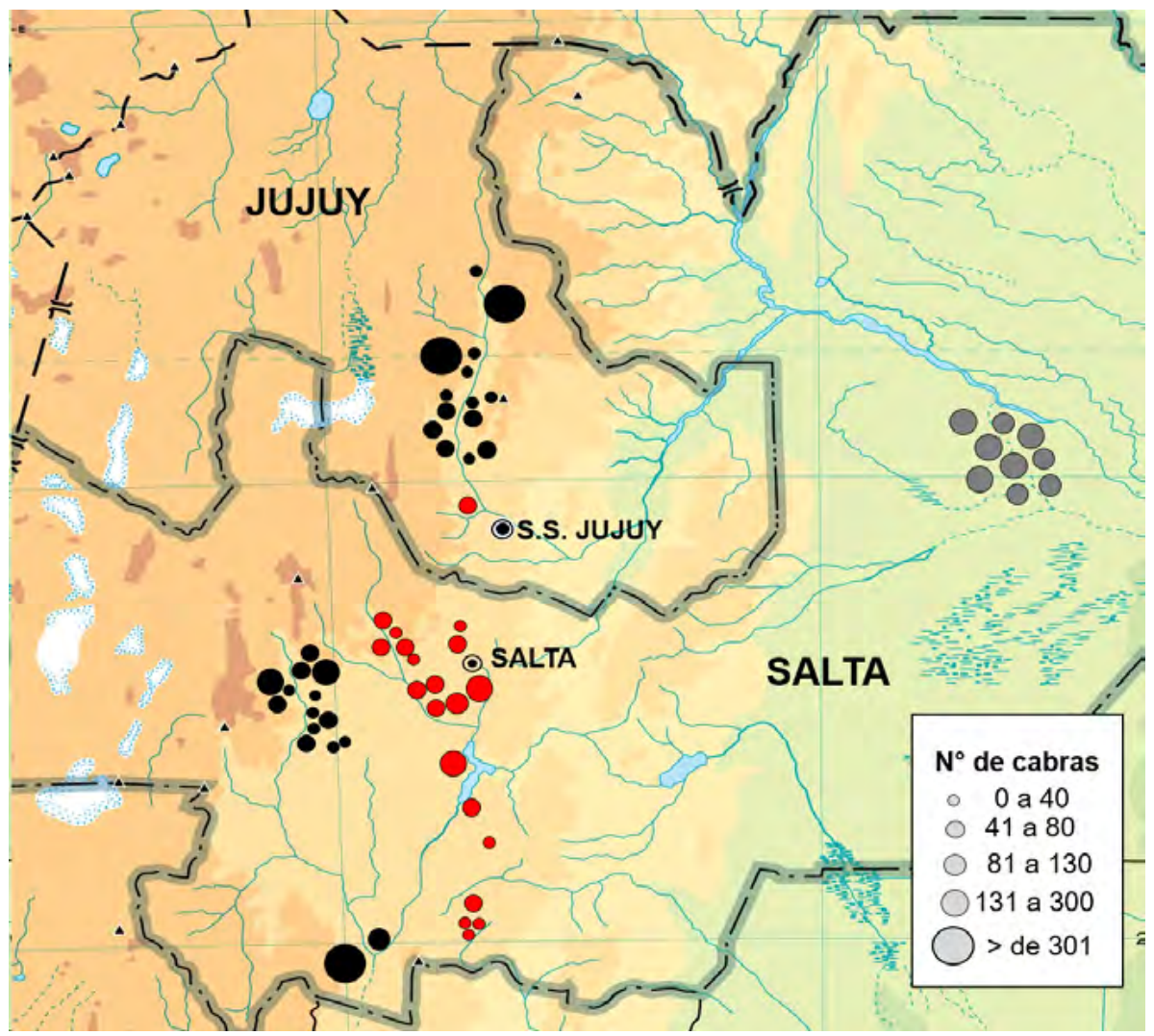

Figura 1: Ubicación de las unidades de producción caprina visitadas y tamaño de las majadas en cuanto al número de cabras en servicio. Majadas ubicadas en la ecorregión de valles y quebradas áridas (círculos negros), del chaco semiárido (círculos grises) y de valles templados y pastizal serrano (círculos rojos). 


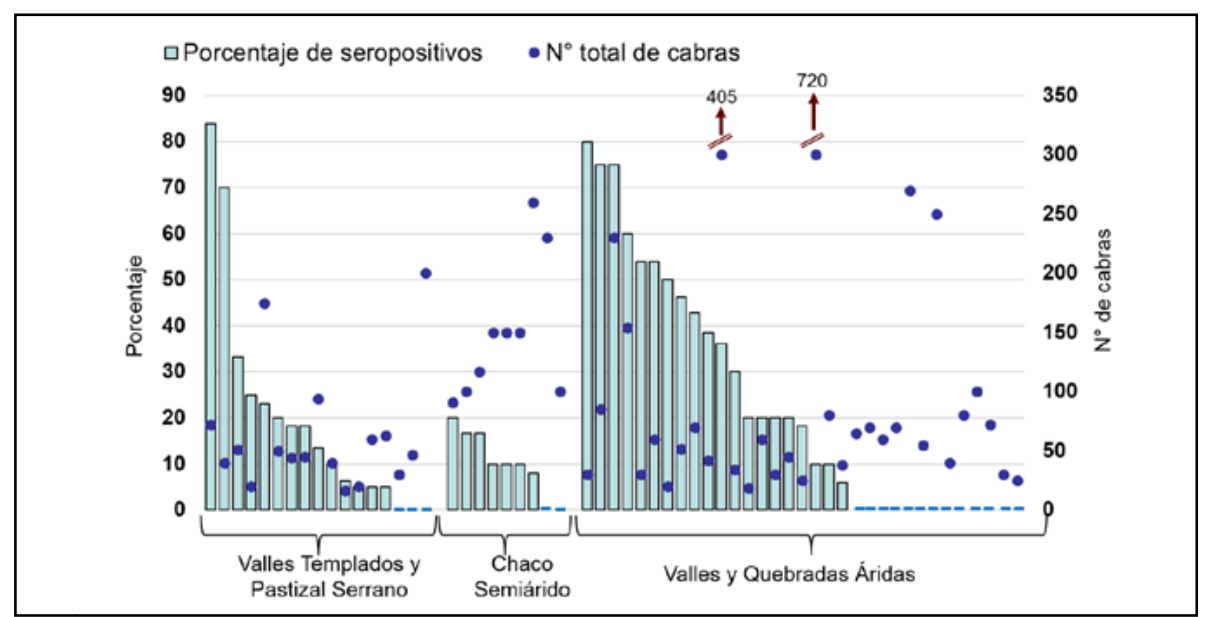

Figura 2: Porcentaje de seropositivos y número total de cabras en servicio de las majadas encuestadas discriminadas por ecorregiones.

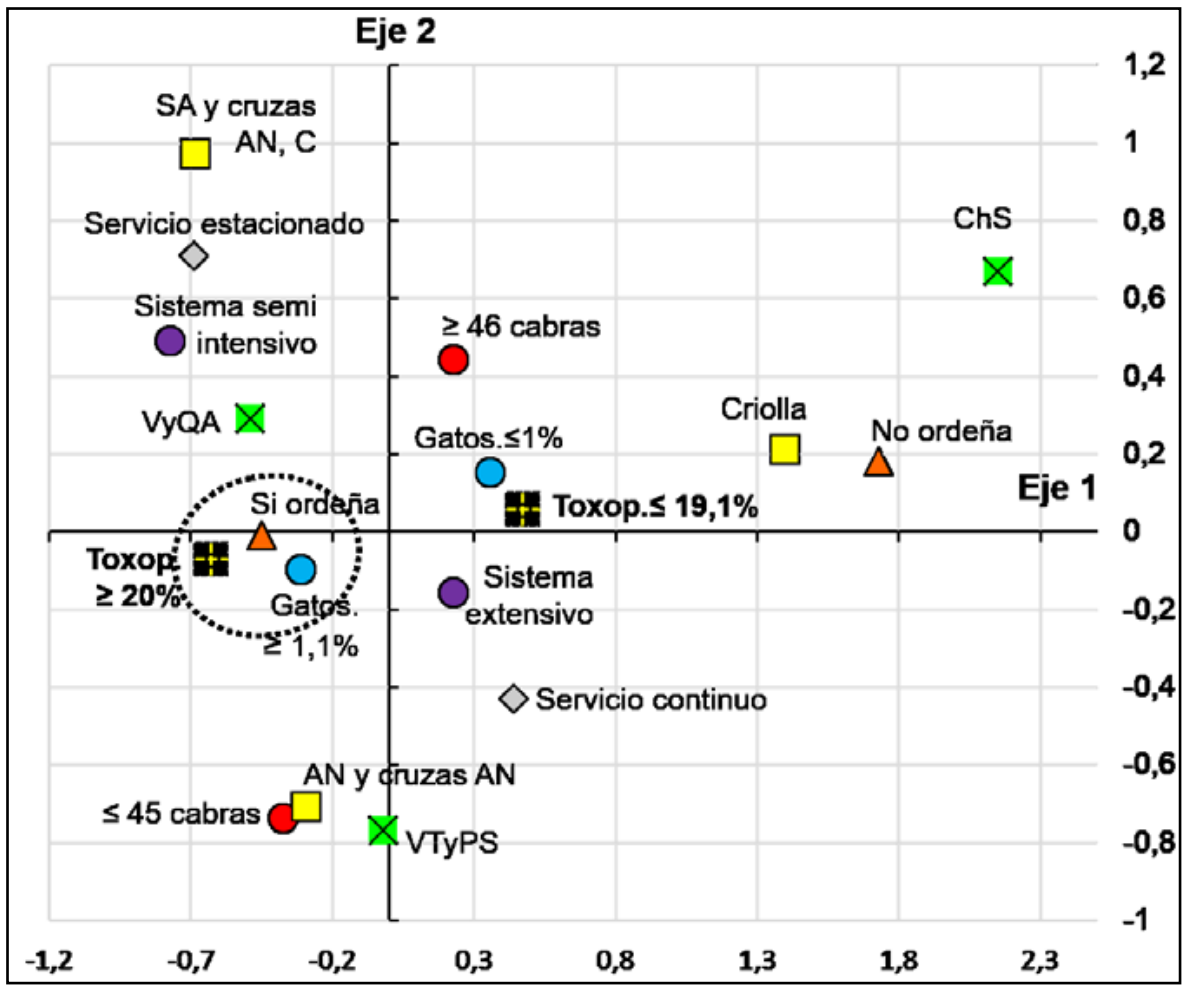

Figura 3: Análisis multivariado de correspondencias múltiples. VyQA: valles y quebradas áridas; CS: chaco semiárido: CS; VTyPS: valles templados y pastizal serrano; SA: Saanen; AN: Anglo Nubian; C: Criollo; Toxop: Porcentaje de seropositivos a T. gondii. Toxop: seroprevalencia de T. gondii 